Meta

Journal des traducteurs

Translators' Journal

\title{
La Constitution canadienne en traduction : quelques pistes de réflexion
}

\section{Ravi J. Gunnoo}

Volume 50, numéro 4, décembre 2005

Pour une traductologie proactive - Actes

For a Proactive Translatology - Proceedings

Por una traductología proactiva - Actas

URI : https://id.erudit.org/iderudit/019919ar

DOI : https://doi.org/10.7202/019919ar

Aller au sommaire du numéro

Éditeur(s)

Les Presses de l'Université de Montréal

ISSN

0026-0452 (imprimé)

1492-1421 (numérique)

Découvrir la revue

Citer cet article

Gunnoo, R. J. (2005). La Constitution canadienne en traduction : quelques pistes de réflexion. Meta, 50(4). https://doi.org/10.7202/019919ar

\section{Résumé de l'article}

Rédigée originellement en anglais, la Constitution canadienne a ensuite été traduite en français. Ce texte de loi consacre le bilinguisme et le bijuridisme sur lesquels le Canada est fondé et, du même coup, la culture de traduction qui est au coeur de la nation canadienne. Or, bien que cet ensemble législatif bilingue soit la loi suprême du Canada, ni les traductologues ni les constitutionnalistes n'ont réalisé une étude comparée des versions anglaise et française de cette Constitution. Dans le cadre du texte qui suit, nous vous présenterons une analyse traductologique relative à deux articles de la Constitution du Canada. 


\title{
La Constitution canadienne en traduction : quelques pistes de réflexion
}

\author{
RAVIJ. GUNNOO \\ Université de Montréal, Montréal, Canada \\ ravi.jaywantrow.gunnoo@umontreal.ca
}

\begin{abstract}
RÉSUMÉ
Rédigée originellement en anglais, la Constitution canadienne a ensuite été traduite en français. Ce texte de loi consacre le bilinguisme et le bijuridisme sur lesquels le Canada est fondé et, du même coup, la culture de traduction qui est au cœur de la nation canadienne. Or, bien que cet ensemble législatif bilingue soit la loi suprême du Canada, ni les traductologues ni les constitutionnalistes n'ont réalisé une étude comparée des versions anglaise et française de cette Constitution. Dans le cadre du texte qui suit, nous vous présenterons une analyse traductologique relative à deux articles de la Constitution du Canada.
\end{abstract}

\begin{abstract}
Originally written in English, Canada's Constitution has been translated into French. This legislative enactment entrenches bilingualism and bijuralism on which Canada is grounded, and by so doing, it also establishes translation culture at the heart of the Canadian nation. However, even though this bilingual statute is the supreme law of Canada, neither translatologists nor constitutional experts have ventured to undertake a comparative study of the English and French texts of the Constitution. In this paper, we will outline a translation study related to two sections of Canada's Constitution.
\end{abstract}

\section{MOTS-CLÉS/KEYWORDS}

traduction juridique, Constitution Act, 1867, Constitution Act, 1982, droit constitutionnel canadien, dominion

«Produit social [...], la langue est le creuset où se sculpte l'histoire d'un peuple. Les mots qui la composent et le discours qui l'exprime traduisent les valeurs d'un patrimoine commun. »

(Gémar 2002 : 164)

"[...] translators approach legal text as if it were a biblical text, bearing a single sacred message revealed to the reader by a close, literalist transcription into the target language." (Kasirer 2001: 340)

« Le principe selon lequel les deux versions d'un texte législatif ont force de loi, soit la règle d'égale autorité, représente sans aucun doute un concept théorique se situant au cœur de l'interprétation législative bilingue. » (Lavoie 2002 : 199)

\section{Introduction}

Rédigée originellement en anglais (Department of Justice Canada 2001), la Constitution canadienne a ensuite été traduite en français (ministère de la Justice Canada 2001). Ce texte de loi consacre le bilinguisme et le bijuridisme sur lesquels le Canada est fondé et, du même coup, la culture de traduction qui est au cœur de la nation canadienne. Or, bien que cet ensemble législatif bilingue soit la loi suprême du Canada, ni les traductologues ni les constitutionnalistes n'ont réalisé une étude comparée des textes anglais et français de cette Constitution. Selon Rémi Michael Beaupré (1986 : 189), la lecture comparative des deux versions officielles représente, à l'étape initiale, l'unique méthode d'interprétation de la législation bilingue au Canada. Par conséquent, dans le droit fil de 
cette démarche contrastive, nous vous présenterons, dans le texte qui suit, une analyse traductologique de deux articles comprenant cinq problèmes de traduction découverts dans la Constitution du Canada.

\section{Cinq premiers problèmes de traduction dans la Constitution canadienne}

Les cinq premiers problèmes de traduction que nous avons décelés dans la Constitution du Canada se trouvent aux articles 3 et 22 de la Loi constitutionnelle de 1867. Nous avons retenu l'article 3 car, en plus d'être le préambule de la Constitution canadienne qui nous introduit au contexte socioculturel et historico-politique de l'époque, il est aussi l'acte de naissance qui a formalisé l'apparition d'un nouveau pays, en l'occurrence le Dominion du Canada. Nous avons aussi sélectionné l'article 22 car il dépeint les particularités d'une instance essentielle du pouvoir législatif d'alors : le Sénat du Dominion du Canada.

\section{Article 3 de la Loi constitutionnelle de 1867}

Article 3 de la Loi constitutionnelle de 1867

\section{Declaration of Union}

It shall be lawful for the Queen, by and with the Advice of Her Majesty's Most Honourable Privy Council, to declare by Proclamation that, on and after a Day therein appointed, not being more than Six Months after the passing of this Act, the Provinces of Canada, Nova Scotia, and New Brunswick shall form and be One

Dominion under the Name of Canada; and on and after that Day those Three Provinces shall form and be One Dominion under that Name accordingly.

\section{3. Établissement de l'union}

Il sera loisible à la Reine, de l'avis du TrèsHonorable Conseil Privé de Sa Majesté, de déclarer par proclamation qu'à compter du jour y désigné, mais pas plus tard que six mois après la passation de la présente loi, les provinces du Canada, de la NouvelleÉcosse et du Nouveau-Brunswick ne formeront qu'une seule et même Puissance sous le nom de Canada; et dès ce jour, ces trois provinces ne formeront, en conséquence, qu'une seule et même Puissance sous ce nom.

\section{passing : passation}

L'article 3 de la la Loi constitutionnelle de 1867 revêt une importance capitale dans la définition constitutionnelle du Canada. Toutefois, malgré sa portée cruciale sur les plans socioculturel et historico-politique, la traduction française de cet article recèle deux flagrantes anomalies. Les deux termes qui posent ici problème sont passing traduit par passation et dominion rendu en français par puissance. Examinons, pour commencer, le mot passing. D'après Cyrille Goulet, passing signifie « the process by which a bill obtains Parliamentary approval and becomes law » (1998: 158). En d'autres termes, le substantif passing désigne le processus par lequel un projet de loi est adopté par le Parlement et devient une loi. Pour Sir David M. Walker, passing veut dire « carrying into law » (1980 : 1286), c'est-à-dire adopter en loi. Par contre, le concept passation, traduction française de passing, ne veut nullement dire adoption d'une loi. En fait, dans le domaine de la comptabilité et de la gestion financière, passation a pour synonymes attribution, octroi ou adjudication. Dans cette optique, passation a pour sens « détermination d'un titulaire d'un marché à la suite du choix de l'offre considérée comme la plus avantageuse » (Ménard et al 2004 : 239). 
Par ailleurs, le terme passation renferme trois acceptions : en droit, il indique l'action de passer, de dresser un contrat ou une écriture comptable; en didactique, passation désigne l'action de passer un examen ou une épreuve à temps limité; et dans la langue courante, lorsque l'on parle de passation des pouvoirs, on fait allusion à la transmission des pouvoirs d'une personne à une autre, d'une institution à une autre. Comme nous pouvons clairement le constater, passation ne se rapporte en aucun cas au processus par lequel un projet de loi est adopté pour devenir une loi. Or, dans la Loi constitutionnelle de 1867, passing a été traduit par passation, terme qui ne dénote pas du tout la réalité conceptuelle de passing. D'après Marie-Éva de Villers (2003 : 1069), passation est un anglicisme du mot anglais passing. Par conséquent, passation est une tournure fautive qu'il faut proscrire lorsqu' on l'emploie pour désigner le processus par lequel une autorité formule et met en vigueur une loi. Traduire passing par passation équivaut donc à un anglicisme, c'est-à-dire une locution propre à la langue anglaise. C'est pourquoi nous proposons de corriger cette erreur en traduisant passing par adoption.

\section{dominion : puissance}

Étudions maintenant le terme dominion, le deuxième problème de traduction identifié à l'article 3 de la Loi constitutionnelle de 1867. Selon Günther Haensch, dominion renvoie à un « selfgoverning, independent member of a commonwealth which maintains some ties with the mother country » (1965 : 214). Sidney I. Landau, quant à lui, définit dominion comme « the land that belongs to a ruler » $(2000: 255)$. Elizabeth E. Martin, de son côté, explique qu'un dominion correspond à un « self-governing nation of the Commonwealth of Nations other than the United Kingdom that acknowledges the British monarch as chief of state » (1990:128). Pour Charles T. Onions, un dominion est un « wholly self-governing or virtually self-governing state of the British Empire or British Commonwealth, particularly one which reached the stage of constitutional development in the late $19^{\text {th }}$ and early $20^{\text {th }}$ centuries » $(1966: 283)$.

Dans les termes de Hubert Reid, un dominion correspond au « mot anglais désignant les anciennes colonies britanniques ayant obtenu une certaine autonomie politique sans avoir toutefois acquis définitivement leur indépendance. La plupart des anciens dominions du Royaume-Uni de Grande-Bretagne sont devenus par la suite membres du Commonwealth [...] » (2004 : 193). D'après Paul Augé, la notion dominion est traduite telle qu'elle en français. À cet égard, le mot anglais dominion, terme de droit constitutionnel, de droit international et de sciences politiques, est normalisé par dominion en français. Nous avons là un bel exemple d'emprunt à l'anglais et ce n'est pas un emploi fautif. À l'instar de common law et equity traduits par common law et equity en français, le concept anglais dominion est en fait devenu l'usage normatif en français. C'est la raison pour laquelle il est rendu tel quel en français.

De plus, selon Paul Augé (1972 : 3349), le mot dominion s'appliquait originellement à tout territoire reconnaissant la souveraineté britannique. Lorsque les colonies canadiennes furent fédérées et dotées d'un gouvernement autonome en 1867, le nouvel État ainsi créé fut appelé « Dominion du Canada ». En 1900, la nouvelle Fédération australienne et la Nouvelle-Zélande accédèrent à leur tour à l'autonomie, et, en 1907, les divers territoires pourvus d'un gouvernement responsable obtinrent le droit d'être distingués dans la terminologie officielle comme « dominions autonomes » et non comme « puissances autonomes ». Dès lors, le terme « dominion » ne s'appliqua plus pratiquement qu'à ces derniers, c'est-à-dire des dominions autonomes. Ceux-ci passèrent progressivement de l'autonomie à l'indépendance, et le Statut de Westminster de 1931 les définit comme des États souverains, égaux en droits, librement associés dans une Communauté de nations, le droit de sécession leur étant expressément reconnu. Ainsi, le concept dominion en français se rapporte à une ancienne colonie britannique de peuplement européen, pourvue d'un gouvernement responsable et démocratique, aujourd'hui État politiquement indépendant au sein du Commonwealth. Depuis 1947, l'emploi du terme «dominion » diminue au profit de l'expression « membre du Commonwealth ou État du Commonwealth ». Par contre, les dictionnaires juridiques ne font aucunement mention de la notion puissance pour désigner le concept dominion. Et d'ailleurs, tout au long de nos recherches terminologiques, nous n'avons rencontré aucune 
équivalence du mot puissance à dominion dans les domaines du droit public, du droit constitutionnel, du droit international et des sciences politiques.

Pourtant, dans la Loi constitutionnelle de 1867, on a traduit dominion par puissance au lieu de garder le terme anglais tel quel. Cela nous amène à nous questionner sur les acceptions de puissance dans le vocabulaire de spécialité. Ce terme possède des sens très précis dans les domaines des mathématiques, de la pharmacologie, de la biotechnologie, de la biochimie, des mesures de grandeurs électriques, des recherches et prospections géologiques, des armes nucléaires, et de la métrologie. Toutefois, le mot puissance ne désigne pas des réalités conceptuelles en droit. Par conséquent, traduire dominion par puissance équivaut à un problème de faux sens en traduction. C'est la raison pour laquelle il faudrait gardait le mot anglais dominion tel quel en français.

Deux autres questions demeurent cependant en suspens : quelle est l'origine du mot dominion et pourquoi l'a-t-on traduit par puissance dans la Loi constitutionnelle de 1867 ?

\section{Origine du concept dominion ${ }^{1}$}

Lors de la Conférence de Charlottetown (1846) portant sur les débats d'une union politique, Sir John A. Macdonald, qui deviendra plus tard le premier premier ministre du Canada, parle de « founding a great British monarchy, in connection with the British Empire. » Les dignitaires du Colonial Office à Londres s'opposent à une telle référence pour nommer un nouveau pays et ils la qualifient de « premature » et de «pretentious ». Le gouvernement britannique craint aussi que cette dernière désignation offense les Américains et ne veut surtout pas, après les tensions de la guerre de Sécession, éveiller leur hostilité.

Afin d'éviter un tel antagonisme, Sir Samuel Leonard Tilley, en s'inspirant de la Bible, choisit le terme Dominion pour désigner le Canada comme un « self-governing colony of the British Empire (the first time it would be so used in reference to a country) while still acknowledging political Dominion was an old British term for a type of government used in New England, and presumably resurrected for new purposes ». Cette appellation est formalisée en 1867 au moment de la Confédération : le British North America Act, 1867 (section 3) crée « One Dominion under the Name of Canada » traduit en français par le faux sens « une seule et même Puissance sous le nom de Canada » (Acte de l'Amérique du Nord britannique, 1867, article 3). Selon l'usage, le mot « dominion » dans « Dominion of Canada » est traduit en français par l'emprunt au terme anglais dominion. La langue française ne dispose pas d'une expression équivalente. De plus, la notion dominion de même que la devise du Canada - A Mari usque ad Mare (From Sea to Sea; D'un océan à l'autre) - proviennent de trois passages de la Bible : en anglais, «He shall have dominion also from sea to sea, and from the river unto the ends of the earth»; en français, « Son règne et son empire s'étendront aussi d'un océan à l'autre, du fleuve

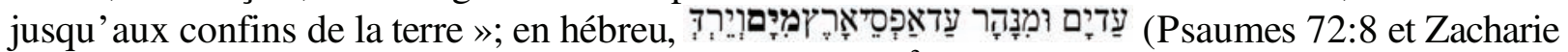
9:10). Par ailleurs, selon le Online Etymology Dictionary ${ }^{2}$ conçu par Harper-Collins, voici la généalogie linguistique de « dominion » :

Dominion: from Middle English “dominioun”, from Old French "dominion”, from Medieval Latin "dominionem" or "dominio", from Latin "dominium" (property, ownership) or

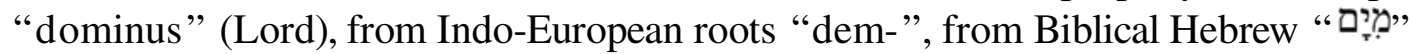
(transliteration: demiyam) meaning divine rule, dominance through spiritual, theocratic and legal authority; British sovereign colonies often were called dominions, hence the Dominion of Canada, the formal title after the 1867 Union, and Old Dominion, the popular name for the U.S. State of Virginia, first recorded 1778.

Avant d'analyser l'article 22, répondons à notre deuxième question demeurée en suspens : pourquoi a-t-on traduit dominion par puissance dans la Loi constitutionnelle de 1867?

\section{De dominion à puissance : dans les coulisses d'un faux sens en traduction}

Selon le Dictionnaire de biographie canadienne en ligne ${ }^{3}$, c'était Eugène-Philippe Dorion avocat, traducteur et homme de lettres - qui a traduit l'Acte de l'Amérique du Nord britannique, 1867 (de nos jours connu comme la Loi constitutionnelle de 1867). Né à Saint-Ours le 6 août 1830 
du mariage entre le docteur Jacques Dorion et Catherine-Louise Lovell, il est décédé à Ottawa le $1^{\text {er }}$ juillet 1872 et inhumé à Saint-Ours. Eugène-Philippe Dorion fit ses études secondaires au séminaire de Saint-Hyacinthe où il entra en 1841. Admis au barreau le 5 décembre 1853, il devenait, deux ans plus tard, traducteur à l'Assemblée législative de la province du Canada. En 1859, il est appelé à y diriger le bureau des traducteurs français, poste qu'il remplit ensuite à Ottawa auprès de la Chambre des communes. Ses contemporains ont vanté ses connaissances des langues mortes, de l'anglais, du français et de quelques langues indiennes. Dorion améliora considérablement le texte français des lois, mais dut parfois se plier devant la volonté des hommes politiques. C'est ainsi que Sir George-Étienne Cartier, co-premier ministre du Canada avec Sir John A. Macdonald, lui aurait imposé la traduction de dominion par «puissance » dans l'Acte de l'Amérique du Nord britannique, 1867 (de nos jours appelé Loi constitutionnelle de 1867).

Dans les coulisses de ce faux sens en traduction émerge un ordre politique délibéré de Sir George-Étienne Cartier - le demandeur officiel de la traduction - à rendre dominion par puissance. Nous avons là un exemple frappant de «translation skopos » (Nord 1991; Vermeer 2000), phénomène dans lequel la fonction du texte cible (le skopos) demeure le facteur déterminant qui influence une traduction. Découlant des directives données par le demandeur, le skopos est un « more or less explicit description of the prospective target situation » (Nord 1991 : 8), imposé sur le traducteur qui n'est plus libre de décider quelle fonction il attribuera au texte cible. Apparait alors un conflit éthique entre l'intention du demandeur et l'expertise du traducteur. Dans cette optique, le demandeur (Sir George-Étienne Cartier) a obligé le traducteur (Eugène-Philippe Dorion) à rendre dominion par puissance en appliquant une « domesticating method » à la traduction, c'est-à-dire, « an ethnocentric reduction of the foreign text to target-language cultural values, bringing the author back home » au lieu d'une « foreignizing method » à la traduction, c'est-à-dire, « an ethnodeviant pressure on those values to register the linguistic and cultural difference of the foreign text, sending the reader abroad » (Venuti $1995: 20$ ). Par conséquent, en raison de cette traduction assimilatrice («domesticating translation ») - dominion : puissance - le traducteur Eugène-Philippe Dorion devient la démonstration vivante de l'invisibilité du traducteur, an illusionism produced by fluent translating, the translator's invisibility at once enacts and masks an insidious domestication of foreign texts, rewriting them in the transparent discourse that prevails in English and that selects precisely those foreign texts amenable to fluent translating. (Venuti 1995: 16-17)

La traduction de dominion par puissance effectuée par Dorion dévoile une distorsion et une perte de sens considérable. En 1867, le concept source dominion était enraciné dans un contexte linguistique, textuel et culturel complexe. Le sens de dominion, son intention communicative et ses répercussions interprétatives découlaient de la relation qu'entretenait cette notion avec son contexte. Cela devait être un exercice périlleux que de faire sortir dominion de son environnement contextuel et de le recréer comme puissance dans une écologie linguistique étrangère. Eugène-Philippe Dorion a déraciné dominion de sa toile de fond contextuelle en essayant vaillamment de transplanter le sens fragile que véhiculait cette expression. Cependant, il a échoué dans sa tentative car, comme nous l'avons démontré plus haut, le résultat de sa traduction - le translatum (Vermeer 2000) - a été un faux sens. Alors, pourquoi Sir George-Étienne Cartier a-t-il choisi puissance comme équivalent à dominion et pourquoi l'a-t-il imposé sur Dorion? Était-ce pour des raisons politiques délibérées? Cartier essayait-il de hisser la nouvelle confédération canadienne vers le statut d'une « puissante » monarchie britannique au sein de l'Empire du Royaume-Uni de Grande Bretagne et d'Irlande? Combien « puissant » était le Dominion du Canada pendant les jours, les mois et les années qui ont succédé à la Confédération? Le Dominion du Canada était-il une « puissance » militaire, économique ou politique?, Malheureusement, les historiens du Canada n'ont pas consigné la raison pour laquelle Sir George-Étienne Cartier a pris une telle décision. 


\section{Article 22 de la Loi constitutionnelle de 1867}

Arrêtons-nous maintenant à l'article 22 de la Loi constitutionnelle de 1867, article dans lequel nous avons découvert trois autres problèmes de traduction.

Article 22 de la Loi constitutionnelle de 1867

\section{Representation of}

Provinces in Senate

In relation to the Constitution of the Senate

Canada shall be deemed to consist of Four

Divisions:

Ontario;

Quebec;

The Maritime Provinces, Nova Scotia and

ew Brunswick, and Prince Edward Island;

The Western Provinces of Manitoba,

British Columbia, Saskatchewan, and Alberta;

which Four Divisions shall (subject to the

Provisions of this Act) be equally represented in the Senate as follows: Ontario by twenty-four senators; Quebec by twenty-four senators; the Maritime Provinces and Prince Edward Island by twenty-four senators, ten thereof representing Nova Scotia, ten thereof representing New Brunswick, and four thereof representing Prince Edward Island; the Western Provinces by twentyfour senators, six thereof representing Manitoba, six thereof representing British Columbia, six thereof representing Saskatchewan, and six thereof representing Alberta; Newfoundland shall be entitled to be represented in the Senate by six members; the Yukon Territory and the Northwest Territories shall be entitled to be represented in the Senate by one member each.

In the Case of Quebec each of the Twenty-four Senators representing that Province shall be appointed for One of the Twenty-four Electoral

Divisions of Lower Canada specified in Schedule A. to Chapter One of the Consolidated Statutes of Canada.

\section{Représentation des} provinces au Sénat

En ce qui concerne la composition du Sénat, le Canada sera censé comprendre quatre divisions :

Ontario;

Québec;

les provinces Maritimes - la Nouvelle-Écosse et le Nouveau-Brunswick ainsi que l'Île-du-PrinceÉdouard;

les provinces de l'Ouest : le Manitoba, la Colombie-Britannique, la Saskatchewan et l'Alberta;

les quatre divisions doivent (subordonnément aux révisions de la présente loi) être également représentées dans le Sénat, ainsi qu'il suit: Ontario par vingt-quatre sénateurs; Québec par vingt-quatre sénateurs; les Provinces maritimes et l'Île-du-PrinceÉdouard par vingt-quatre sénateurs, dont dix représentent la Nouvelle-Écosse, dix le NouveauBrunswick, et quatre l'Île-du-Prince-Édouard; les Provinces de l'Ouest par vingt-quatre sénateurs, dont six représentent le Manitoba, six la ColombieBritannique, six la Saskatchewan et six l'Alberta; la province de Terre-Neuve aura droit d'être représentée au Sénat par six sénateurs; le territoire du Yukon et les territoires du Nord-Ouest ont le droit d'être représentés au Sénat par un sénateur chacun.

En ce qui concerne la province de Québec, chacun des vingt-quatre sénateurs la représentant, sera nommé pour l'un des vingt-quatre collèges électoraux du Bas-Canada énumérés dans la cédule A, annexée au chapitre premier des statuts refondus du Canada.

\section{Arrière-plan textuel de l'article 22 de la Loi constitutionnelle de 1867}

L'article 22 - consacré à la représentation des provinces au Sénat - se trouve entre la troisième partie ( «Pouvoir exécutif ») et la cinquième partie («Constitutions provinciales) de la Loi constitutionnelle de 1867. Cet article est plus précisément situé dans la quatrième partie intitulée «Pouvoir législatif », laquelle est subdivisée en trois sections : «Le Sénat», «La Chambre des Communes » et «Législation financière; sanction royale ». Le corps de l'article 22 est structuré en quatre segments : un énoncé d'ordre général, une énumération relative à cet énoncé, une décomposition chiffrée de cette énumération, et une précision ponctuelle pour une province en particulier. Nous distinguons les aspects suivants à propos de ces quatre segments de l'article 22 : 
énoncé général - « En ce qui concerne la composition du Sénat, le Canada sera censé comprendre quatre divisions [...] »; énumération de l'énoncé - «1. Ontario; 2. Québec; 3. les provinces Maritimes - la Nouvelle-Écosse et le Nouveau-Brunswick ainsi que l'Île-du-Prince-Édouard; 4. les provinces de 1'Ouest : le Manitoba, la Colombie-Britannique, la Saskatchewan et l'Alberta; »; décomposition chiffrée de l'énumération - «les quatre divisions doivent [...] être également représentées dans le Sénat, ainsi qu'il suit : Ontario par vingt-quatre sénateurs; Québec par vingtquatre sénateurs; les Provinces maritimes et l'Île-du-Prince-Édouard par vingt-quatre sénateurs, dont dix représentent la Nouvelle-Écosse, dix le Nouveau-Brunswick, et quatre l'Ille-du-Prince-Édouard $[\ldots] »$; et une précision ponctuelle - «En ce qui concerne la province de Québec, [...]».

\section{provisions : révisions; electoral divisions : collèges électoraux; schedule : cédule}

La traduction française, quant à elle, de l'article 22 de la Loi constitutionnelle de 1867 renferme trois anomalies. Les trois notions qui posent ici problème sont provisions rendu par révisions, electoral divisions traduit par collèges électoraux, et schedule exprimé par cédule. Considérons d'abord le terme provisions. Selon Henry C. Black, provisions, dont les variantes synonymiques sont clauses, paragraphs et stipulations, renvoie aux acceptions suivantes en anglais :

Provision: A single clause, paragraph, stipulation or subdivision of a pleading or legal document, such as a contract, deed, will, constitution, or statute. It is sometimes a sentence or part of a sentence. (1979: 1102) Clause: A separate section of a legal document, such as a statute, contract, will, deed, or constitution. (1979 : 226) Paragraph: A part or section of a statute, pleading, affidavit, will, trust, constitution, etc, which contains one article, the sense of which is complete. (1979: 1001) Stipulation: A material condition, requirement, provision or article in an agreement. (1979:1269)

D'après ces acceptions, la notion anglaise provision ne désigne nullement le terme français révision. En fait, à quoi correspond révision dans la langue de spécialité et dans le vocabulaire juridique? Dans le jargon spécialisé, révision véhicule des sens précis dans au moins deux domaines. En vérification comptable, cette notion se rapporte au « contrôle effectué par un professionnel compétent et indépendant en vue d'exprimer une opinion motivée sur la régularité et la sincérité des comptes, ainsi que sur la fidélité de l'image qu'ils donnent du patrimoine, de la situation financière et des résultats de l'entité en cause [...] » (Ménard et al 2004 : 85). En correction d'épreuves, révision dénote « une vérification et une mise au point de la copie qui, lorsqu'elle a lieu, précède la préparation technique de cette même copie. » (Paradis 1993 : 496) Dans le vocabulaire juridique, Gérard Cornu nous offre les éclaircissements ci-dessous sur le terme révision :

1 Réexamen d'un corps de règles en vue de son amélioration. Ex. révision constitutionnelle, révision législative. 2 Modification d'un acte juridique [...] en vue de son adaptation aux circonstances. 3 Réexamen juridictionnel d'une décision en vue de sa rétractation [...] ou de son annulation par une juridiction supérieure. 4 Mise à jour (en général périodique) d'un acte, d'une loi [...]. (2000 : 711-712)

Ainsi, lorsque l'on parle d'amendement, de révision ou de modification constitutionnelle, on fait allusion à la procédure par laquelle le contenu d'une constitution est modifié selon les dispositions prévues par la constitution en vigueur. En outre, selon Olivier Duhamel et Yves Mény,

[...] la doctrine distingue les constitutions souples pour lesquelles la révision est relativement aisée et les constitutions rigides pour lesquelles la procédure de révision s'avère plus difficile. Dans cette dernière hypothèse, la plus fréquente désormais, il existe en général des conditions de majorité particulière, des délais spécifiques ou le recours à des instances de ratification ad hoc. L'idée de base consiste à estimer qu'une constitution doit bénéficier d'une garantie de longévité et de stabilité et qu'elle ne doit pas pouvoir être modifiée au gré des majorités politiques. La révision doit être entourée de précautions destinées à souligner l'aspect fondamental du pacte constitutionnel. (1992 : 932) 
À la lumière des recoupements terminologiques ci-dessus, nous constatons que le terme anglais provision ne se rapporte aucunement au mot français révision. D'ailleurs, dans la version anglaise de l'article 22 de la Loi constitutionnelle de 1867 - « [...] which Four Divisions shall (subject to the Provisions of this Act) be equally represented in the Senate as follows [...] traduite par « [...] les quatre divisions doivent (subordonnément aux révisions de la présente loi) être également représentées dans le Sénat, ainsi qu'il suit [...]»- provisions ne signifie pas du tout révisions. Nous nous retrouvons ici devant un cas de faux sens en traduction car révisions ne correspond surtout pas au champ sémantico-juridique de provisions. Pour corriger cette erreur, nous proposons de rendre provisions par dispositions, clauses ou encore stipulations.

\section{electoral divisions : collèges électoraux}

Passons à présent au deuxième problème de traduction de l'article 22 : electoral divisions traduit par collèges électoraux. Cyrille Goulet comprend un electoral division comme « [...] any area whose inhabitants are entitled to elect a member; the body of voters who elect a representative for their area » (1998 : 53). Wayne Cole et Lise Boucher soulignent dans le Elections Glossary Lexique des élections, publié par le Secrétariat d'État du Canada, que electoral division peut être entendu comme constituency, electoral district ou riding et ils le définissent comme « the group of voters belonging to any of the official areas of a country that elect someone to represent them nationally » (1988: 22). De plus, Cole et Boucher recommandent de traduire electoral division par circonscription (pour les élections provinciales au Canada) ou par comté (pour les élections fédérales au Canada).

Par contre, dans l'article 22 de la Loi constitutionnelle de 1867, electoral division a été traduit par collèges électoraux qui n'est rien de moins qu' une traduction littérale du concept anglo-américain electoral college. Ce concept anglo-américain ne rejoint absolument pas les réalités électorales canadiennes puisqu'il reflète surtout les procédures à enclencher pour faire élire le président et le vice-président des États-Unis d'Amérique. À l'inverse du Canada qui est une monarchie constitutionnelle, les États-Unis sont une fédération républicaine conformément au «Article 1V, Section 4 of The Constitution of the United States of America » qui stipule que «The United States shall guarantee to every State in this Union a Republican Form of Government » (Levy and Karst 2000 : 2209). D'où les différences fondamentales dans la terminologie institutionnelle des réalités électorales et du système politique entre le Canada et son grand voisin du Sud. À cet égard, nous nous rallions à Jean-Claude Gémar lorsqu'il précise que

[...] le langage du droit véhicule des notions, des institutions et des procédures qui sont tellement propres à chaque langue et culture juridiques que l'on ne peut les transposer telles quelles d'une langue et d'un système à un autre sans risquer à tout moment l'impropriété, le contresens, voire le non-sens juridiques (Crépeau 1993; Sacco 1999 : 169; Vanderlinden 1999; Viau 2000). Aussi la traduction juridique, tout particulièrement entre le français et l'anglais, pose-t-elle un problème particulièrement aigu dans la mesure où il ne s'agit pas simplement de passer d'une langue à une autre, mais d'un système à un autre (Didier 1990), si différents l'un de l'autre [...]. (2002: 167)

De plus, voici la portée sémantico-constitutionnelle de l'américanisme electoral college qui le distingue du electoral division canadien :

Electoral College: Mechanism by which the president and the vice president are selected. The Electoral College was established by Article II, and it requires states to select electors equal in number to their representation in Congress. These electors cast votes on a date following the presidential election. The electors never meet as a national body, but cast their ballots from their respective states. If no candidate for president receives an absolute majority of electoral votes, the House of Representatives chooses from the top three candidates in total electoral votes. In the House process, each state delegation has a single vote. (Chandler and Enslen 1985 : 597) 
Nous ne pouvons, par conséquent, accepter l'américanisme electoral college (collèges

électoraux) pour le electoral division canadien. Pour rectifier le tir et ainsi rétablir la toile de fond des réalités électorales et du système politique en vigueur sur le territoire du Canada, nous suggérons de traduire electoral division par comté car ce terme reflète bien le découpage de toute localité ou zone territoriale ayant le droit d'élire un député.

\section{schedule : cédule}

Avant de conclure notre article, analysons le troisième problème de traduction repéré à l'article 22 de la Loi constitutionnelle de 1867 : schedule rendu par cédule. Dans les mots de Henry C. Black, un schedule en anglais désigne un « sheet of paper annexed to a statute, deed, deposition, will, contract, constitution, or other instrument, exhibiting in detail the matters mentioned or referred to in the principal document [...]» (1979: 1206). Pour Chandler et Enslen, ce mot dénote «a list or statement of supplementary details appended to another legal document » (1985: 423). Selon Elizabeth E. Martin, schedule signifie « a written statement of details forming an appendix to an act, an agreement, a law [...] » (1990 : 672). Conformément à ces définitions, cédule en français renvoie-t-il au schedule en anglais? Non, tel n'est pas le cas puisque cédule, d'après Gérard Cornu, comporte deux acceptions, l'une en droit civil et l'autre en fiscalité. En droit civil, cédule veut dire un « ancien terme de pratique, très rarement employé, désignant un titre reconnaissant une promesse, un engagement [...]; Ex. cédule hypothécaire : titre constatant l'inscription sur un livre foncier d'une dette sur un immeuble, qui, remis au propriétaire de cet immeuble, est négociable. » (Cornu 2000 : 130) En fiscalité, cédule se rapporte au « terme désignant chaque catégorie d'impôts sur les revenus (dits cédulaires). Ex. cédule des bénéfices industriels et commerciaux. » (Cornu 2000 : 131) En outre, selon Marie-Éva de Villers (2003 : 250), cédule est un anglicisme pour horaire, programme, calendrier. Par conséquent, cédule est un emploi fautif qu'il faut bannir car il est en rupture sémantique avec le schedule anglais.

C'est la raison pour laquelle nous proposons de rendre schedule par annexe, notion qui s'harmonise parfaitement avec les réalités conceptuelles du terme anglais. D' ailleurs, voici les acceptions que donne Gérard Cornu à notre proposition de traduire schedule par annexe :

\footnotetext{
I Sens général. Disposition jointe à un acte (loi, traité, décret, etc.) pour en compléter les énonciations; ex. annexe du Nouveau Code de procédure civile [...]. Pièce jointe à un acte instrumentaire dont elle est l'accessoire, parfois à peine d'irrecevabilité de l'acte principal (ex. la requête en divorce sur demande conjointe doit comprendre en annexe un projet de convention définitive portant règlement des effets du divorce) mais qui, n'ayant pas d'existence indépendante, n'a pas - quant à sa réception par un officier public - à faire l'objet d'un dépôt [...]. II Droit international public. Dispositions jointes à un traité et destinées à régler les détails techniques ou à préciser le sens de certaines dispositions (la valeur juridique des annexes étant précisée par l'accord même). III Droit du travail. Texte joint à une convention collective et concernant en particulier un sujet ou une catégorie de salariés. (2000:56)
}

\section{Conclusion}

Pour terminer, appliquons l'approche comparative de Gideon Toury (1995) à l'exemple de faux sens en traduction que nous avons repéré à l'article 3 de la Loi constitutionnelle de 1867. Dans son approche, Toury propose une démarche permettant d'étudier la traduction en tant que processus et résultat, et de déterminer si la traduction tend vers le texte et la langue sources ou vers le texte et la langue cibles. Cette orientation, délimitée par la relation d'équivalence qui s'établit entre la traduction et son original, peut être formelle ou fonctionnelle. Dans le cas de dominion rendu par puissance, il est évident que le texte ou le terme d'arrivée (le translatum selon Vermeer) produit par Eugène-Philippe Dorion (traducteur de la Loi constitutionnelle de 1867) est un texte ou un terme qui s'oriente vers la langue cible. D'où la relation d'équivalence fonctionnelle qui s'établit entre la notion traduite puissance et le concept original dominion. En examinant le processus qui a conduit à ce translatum (dominion : puissance), nous découvrons qu'il n'y a 
vraisemblablement pas eu de processus de négociation par lequel celui qui commande la traduction (Sir George-Étienne Cartier, co-premier ministre du Dominion du Canada) explique au traducteur (Eugène-Philippe Dorion) le but de la traduction et les conditions dans lesquelles celle-ci doit être réalisée. Dans ce cas, le demandeur de la traduction (Cartier), qui est aussi la figure d'autorité politique puisqu'il incarne la plus haute responsabilité du pouvoir exécutif, a carrément obligé le traducteur Dorion, subalterne exécutant, de se plier à ses quatre volontés et à traduire dominion par puissance.

Se dévoile ici un conflit éthique entre l'intention du demandeur et l'expertise du traducteur. Expert en action traductionnelle, Dorion était le seul responsable de la tâche qui lui avait été confiée et du résultat escompté. Ainsi, une fois que le traducteur connait bien la fonction du texte d'arrivée, le texte de départ devient une partie de sa tâche et le point de repère à partir duquel il établira la hiérarchie des éléments qui façonneront le texte cible. Cependant, tel n'a pas été le cas dans la traduction de dominion par puissance. Le traducteur Dorion, directeur du bureau des traducteurs français de la Chambre des communes à Ottawa, n’était plus libre de décider quelle fonction il attribuerait au texte cible même s'il était expert en la matière. De plus, en tant qu'employé travaillant pour une prestigieuse institution gouvernementale, le traducteur subalterne Dorion devait se soumettre à l'autorité politique et aux désidératas de Cartier, le chef du pouvoir exécutif de l'époque. Dorion devait se courber même s'il était peut être en désaccord avec le choix terminologique de son premier ministre. Dorion se devait d'être invisible afin d'accepter et de produire le concept puissance - une traduction fluide, transparente, une traduction imposée par Cartier et qui évacue les particularités étymologique, linguistique et stylistique du terme étranger dominion. Par conséquent, cette soumission a fait de Dorion le traducteur invisible et effacé de la Loi constitutionnelle de 1867. Comme l'affirme à juste titre Venuti, au nom de cette fameuse invisibilité, le traducteur est forcé de se transformer en subtil manipulateur des textes, des langues, des concepts, des cultures et de l'histoire :

\footnotetext{
Invisibility is the term I will use to describe the translator's situation and activity in contemporary AngloAmerican culture. It refers to two mutually determining phenomena: one is an illusionistic effect of discourse, of the translator's own manipulation of English; the other is the practice of reading and evaluating translations that has long prevailed in the United Kingdom and the United States, among other cultures, both English and foreign-language. A translated text, whether prose or poetry, fiction or nonfiction, is judged acceptable by most publishers, reviewers, and readers when it reads fluently, when the absence of any linguistic or stylistic peculiarities makes it seem transparent, giving the appearance that it reflects the foreign writer's personality or intention or the essential meaning of the foreign text - the appearance, in other words, that the translation is not in fact a translation, but the "original". The illusion of transparency is an effect of fluent discourse, of the translator's effort to insure easy readability by adhering to current usage, maintaining continuous syntax, fixing a precise meaning. What is so remarkable here is that this illusory effect conceals the numerous conditions under which the translation is made, starting with the translator's crucial intervention in the foreign text. The more fluent the translation, the more invisible the translator, and, presumably, the more visible the writer or meaning of the foreign text. (Venuti $1995: 1-2$ )
}

Au terme de cet article, nous ne pouvons nous empêcher de donner raison à Louis-Philippe Pigeon, ancien juge à la Cour suprême du Canada, qui écrivit qu'avant la promulgation de la Loi sur les langues officielles en 1969, les lois « [...] étaient publiées dans les deux langues, mais la rédaction en était unilingue et la traduction servile » (1982:273). Toutefois, les observations que nous avons faites sur les deux difficultés analysées dans le présent article ne sont pas, à ce stade de notre étude, représentatives de l'ensemble des lois constitutionnelles du Canada. Nos recherches à venir nous permettront, d'abord, de mieux baliser notre nouvelle démarche interdisciplinaire qui fait le va-et-vient entre la traductologie et le droit constitutionnel canadien. Ces mêmes recherches nous aideront, ensuite, à mieux appréhender, sous l'angle de la traduction, ce qu'est le Canada - « [...] un État juridiquement, institutionnellement et linguistiquement complexe dont l'intérêt principale se trouve dans la réconciliation de ces régimes juridiques multiples. »(Cadiet 2004 : 143) 


\section{NOTES}

1. Consultez l'Encyclopédie canadienne à

http://www.thecanadianencyclopedia.com/index.cfm?PgNm=ArchivedFeatures\&TCE_Version=F\&FeatureId=11 et

ANSWERS.COM à http://www.answers.com/topic/canada-s-name.

2. Online Etymology Dictionary: http://www.etymonline.com/index.php?search=dominion\&searchmode=or

[Consulté le 28 octobre 2005]

3. DICTIONNAIRE DE BIOGRAPHIE CANADIENNE EN LIGNE.

http://www.biographi.ca/FR/ShowBio.asp?BioId=39074\&query=dorion [Consulté le 23 octobre 2005]

\section{RÉFÉRENCES}

Augé, P. (dir.) (1972) : Grand Larousse encyclopédique, tome 5, Paris, Librairie Larousse.

BEAUPRÉ, R. M. (1986) : Interprétation de la législation bilingue, traduit de l'anglais par Ethel GroffierAtala, Montréal, Wilson \& Lafleur.

Black, H. C. (1979): Black's Law Dictionary: Definitions of the Terms and Phrases of American and English Jurisprudence, Ancient and Modern, $5^{\text {th }}$ ed., St. Paul (Minnesota), West Publishing Co.

CAdiet, L. (dir.) (2004) : Dictionnaire de la justice, Paris, Presses universitaires de France.

Chandler, R. C., Enslen, R. A. and P. G. Renstrom (eds) (1985): The Constitutional Law Dictionary, Volume 2: Governmental Powers, Santa Barbara (California), ABC-CLIO Information Services.

Cole, W. et L. Boucher (1988): Elections Glossary - Lexique des élections, $2^{\text {nd }}$ ed., Ottawa,

Department of the Secretary of State of Canada/Secrétariat d'État du Canada.

Cornu, G. (dir.) (2000) : Vocabulaire juridique, 8 éd., rev. et augm., Paris, Presses universitaires de

France.

CRÉPEAU, P. A. (1993) : «L'affaire Daigle et la Cour suprême du Canada ou la méconnaissance de la tradition civiliste », in Ernest CAPARROS dir. Mélanges Germain Brière, Montréal, Wilson and Lafleur, p. 217-281.

DEPARTMENT OF JUSTICE CANADA (2001): Consolidation of the Constitution Acts, 1867 to 1982 , Ottawa, Canadian Government Publishing/Public Works and Government Services Canada.

DIDIER, E. (1990) : Langues et langages du droit, Montréal, Wilson \& Lafleur.

DE VILLERS, M.-É. (2003) : Multidictionnaire de la langue française, 4éd., Montréal,

Québec/Amérique.

Duhamel, O. et Y. MÉNY (1992) : Dictionnaire constitutionnel, $2^{\mathrm{e}}$ éd., Paris, Presses universitaires de

France.

GÉMAR, J.-C. (2002) : « Le plus et le moins-disant culturel du texte juridique. Langue, culture et

équivalence », META 47-2, p. 163-176.

Goulet, C. (1998) : Vocabulary of Parliament - Vocabulaire du Parlement, éd. rev. et augm., Ottawa, Travaux publics et Services gouvernementaux Canada/Bureau de la traduction du Canada.

HAENSCH, G. (1965): Dictionary of International Relations and Politics, New York, Elsevier.

KASIRER, N. (2001) : « François Geny’s libre recherche scientifique as a Guide for Legal Translation », Louisiana Law Review 61-2, p. 331-352.

LandaU, S. I. (ed.) (2000): Cambridge Dictionary of the American Constitution, Cambridge, Cambridge University Press.

LAvoie, J. (2002) : «Le discours sur la traduction juridique au Canada », META 47-2, p. 198-210.

LEVY, L. W. and K. L. KARST (eds) (2000): Encyclopedia of the American Constitution, $2^{\text {nd }}$ ed., New

York/London, Macmillan Reference USA.

MARTIN, E. E. (ed) (1990): A Concise Dictionary of Law, Oxford, Oxford University Press.

MÉNARD, L. et H. OLIVIER (2004) : Dictionnaire de la comptabilité et de la gestion financière : anglais-

français avec index français-anglais, $2^{\mathrm{e}}$ éd., Toronto, Institut canadien des comptables agréés.

MINISTÈRE DE LA JUSTICE CANADA (2001) : Codification administrative des Lois

constitutionnelles de 1867 à 1982, Ottawa, Éditions du gouvernement du Canada/Travaux publics et

Services gouvernementaux Canada. 
NORD, C. (1991): Text Analysis in Translation: Theory, Methodology and Didactic Application of a Model for Translation-Oriented Text Analysis, Amsterdam, Atlanta, Rodopi.

OnIONS, C. T. (ed.) (1966): The Oxford Dictionary of English Etymology, Oxford, Clarendon Press. PARADIS, L. (1993) : Vocabulaire des industries graphiques - Graphic Arts Vocabulary, Ottawa, Secrétariat d'État du Canada/ Department of the Secretary of State of Canada.

PIGEON, L.-P. (1986) : Rédaction et interprétation des lois, $3^{\mathrm{e}}$ éd., Québec, Éditeur officiel du ministère des Communications.

Pilette, L. (1993) : La Constitution canadienne, Montréal, Éditions du Boréal.

REID, H. (2004) : Dictionnaire de droit québécois et canadien, $3^{\mathrm{e}}$ édition, Montréal, Wilson and Lafleur. SACCO, R. (1999) : «Langue et droit » in R. SACCO et L. CASTELLANI (dir.) Les multiples langues du droit européen uniforme, Torino, L'Harmattan, p. 163-185.

Toury, G. (1995): Descriptive Translation Studies and Beyond, Amsterdam, John Benjamins Publishing. VAnderlinden, J. (1999) : «Langue et droit », Contemporary Law, Cowansville (Québec), Les Éditions Yvon Blais, p. 25-68.

VenutI, L. (1995): The Translator's Invisibility: a History of Translation, London; New York, Routledge.

VermeER, H. J. (2000): "Skopos and Commission in Translation Action", The Translation Studies Reader, edited by Lawrence Venuti, London, Routledge, p. 221-232.

VIAU, P. (2000) : «Quelques considérations sur la langue, le droit, le bilinguisme et le bijuridisme au Canada », Langue et droit, Bruxelles, Bruylant, p. 141-151.

Walker, D. M. (1980): The Oxford Companion to Law, Oxford and New York, Oxford University Press. 